\title{
Skyrme Crystal In A Two-Dimensional Electron Gas
}

\author{
L. Brey ${ }^{1}$ H.A.Fertig ${ }^{2}$, R.Côté ${ }^{3}$ and A.H.MacDonald ${ }^{4}$. \\ ${ }^{1}$ Instituto de Ciencia de Materiales (CSIC). Universidad Autónoma C-12, 28049, Madrid, Spain. \\ 2 Department of Physics and Astronomy, University of Kentucky, Lexington, Kentucky 40506-0055. \\ ${ }^{3}$ Département de Physique et Centre de Recherches en Physique du Solide, Université de Sherbrooke, Sherbrooke, Québec, \\ Canada J1K 2R1. \\ 4 Department of Physics, Indiana University, Bloomington, Indiana 47405.
}

(October 17, 2018)

The ground state of a two-dimensional electron gas at Landau level filling factors near $\nu=1$ is a Skyrme crystal with long range order in the positions and orientations of the topologically and electrically charged elementary excitations of the $\nu=1$ ferromagnetic ground state. The lowest energy Skyrme crystal is a square lattice with opposing postures for topological excitations on opposite sublattices. The filling factor dependence of the electron spin-polarization, calculated for the square lattice Skyrme crystal, is in excellent agreement with recent experiments.

PACS number 73.20.Dx

The incompressible [1] ground state of a twodimensional electron gas (2DEG) at Landau level filling factor $\nu=1$ is ferromagnetic. (Here $\nu \equiv N / N_{\phi}$ is the ratio of the number of electrons to the orbital degeneracy of a Landau level; $N_{\phi}=A B / \Phi_{0}=A /\left(2 \pi \ell^{2}\right)$ where $A$ is the area of the system, $\Phi_{0}$ is the magnetic flux quantum, and $\mathrm{B}$ is the magnetic field strength.) Recently it has been shown 22 5] that, in the limit of weak Zeeman coupling, the lowest energy charged excitations of this state are spin-textures known as skyrmions. [6]. Skyrmions are the lowest energy topologically charged spin-texture excitations of the $\mathrm{SU}(2)$ non-linear $\sigma(\mathrm{NL} \sigma)$ model which describes the long wavelength properties of isotropic ferromagnets. The equivalence of physical charge and topological charge in the present system is a consequence [2,5] of the quantum Hall effect [1] and is responsible for the dominating role of skyrmions in determining many physical properties.

Pioneering studies of skyrmions in the quantum Hall regime relied on $\mathrm{NL} \sigma$ models generalized [2,5] to account for Zeeman coupling to the spin and for the Hartree (electrostatic) interactions present when the charge density is non-uniform. States in the NL $\sigma$ model are specified by a space-dependent unit vector which describes the local orientation of the spin magnetic moment. A skyrmion is characterized by the sign of its topological charge, by its size, and by the global orientation of the spin; with no Zeeman or Hartree coupling the energy of an isolated skyrmion is independent of all three. When Zeeman coupling is included the spin moment outside a skyrmion aligns with the field $\left(m_{z}=1\right)$, the spin moment at the center of a skyrmion is oriented in opposition to the field $\left(m_{z}=-1\right)$. The perpendicular component $\left(m_{\perp}\right)$ of the spin-moment, which must be non-zero as $m_{z}$ changes from -1 to 1 , has a vortex at the skyrmion center. The global azimuthal orientation of $m_{\perp}$ is, importantly for the work described here, still arbitrary. Because the spinmoment is reversed in the interior of a skyrmion, Zeeman coupling favors small skyrmions. On the other hand the Hartree self-interaction energy of the skyrmion favors large skyrmions so that an optimal skyrmion size is established. [2] For Zeeman coupling strengths typical of physical systems the estimated skyrmion size is comparable to microscopic lengths, motivating a microscopic approach. Recently [7], using a microscopic Hartree-Fock approximation, we confirmed the main predictions of the fieldtheory approach and obtained an estimate of the number of reversed spins for each (skyrmion) charge added to the system at $\nu=1$. The quantitative agreement between this estimate and subsequent Knight shift measurements [8] appears to provide incontrovertible evidence in favor of the exotic topological nature of the low-energy charge carriers in this system. In this Letter we report on a Hartree-Fock approximation study of the ground state of the 2DEG for $\nu$ near 1 , when interactions between the charged spin-textures, which we will refer to as skyrmions even when distorted by Zeeman and Hartree couplings, become important.

The most important result of our calculation is shown in Fig. 1 where we compare our theoretical results for the spin-polarization $P$ of the two-dimensional electron gas as a function of filling factor with experiment. [8] ( $P$ is the spatial average of $m_{z}$. ) Without interactions the spins are completely aligned by the Zeeman field for $\nu<1$. The polarization is gradually reduced for $\nu>1$, reaching zero for $\nu=2$, since the Pauli exclusion principle forces the occupation of reversed spins. The large slope of $P$ on either side of $\nu=1$ is in sharp contrast with the non-interacting electron result and can be related to the number of reversed spins in an isolated skyrmion. [8,2,7] Our calculations at finite $|\nu-1|$ were performed assuming that the ground state of the system is a crystal of charged skyrmion spin-textures. These states are analogous to the Skyrme crystal states which arise [9] in studies of dense nuclear matter using Skyrme's topological excitation model. The Hartree-Fock equations have many different solutions; all have long-range order in both the spatial arrangement of skyrmion centers and in the azimuthal orientation of $m_{\perp}$ near each skyrmion center. Results are shown for two cases: i) a triangular lattice of skyrmions with identical orientations of $m_{\perp}$ near each 
site (TLF state) and, ii) a square lattice with oppositely directed orientations of $m_{\perp}$ on the two square sublattices (SLA state). (The initials $\mathrm{A}$ and $\mathrm{F}$ in the labels of these states suggest the obvious analogies with ferromagnetic and antiferromagnetic states of spin systems on two-dimensional square and triangular lattices.) We see in Fig. 1 that the slope of the spin-polarization curve decreases in magnitude as we move away from $\nu=1$ in both cases, indicating that the skyrmions shrink as they become more dense. The shrinking is evidently much more rapid for the TLF state than for the SLA state. The results for $P$ for the latter case are in quite reasonable agreement with experiment and indeed, as we discuss below, we find that the square Skyrme lattice has lower energy than the triangular Skyrme lattice except at very small $|\nu-1|$. Comparison between theory and experimental results for $P$ suggests that the ground state is a square Skyrme crystal until $|\nu-1| \approx 0.2$.

Our calculations are greatly simplified by working entirely within the lowest orbital Landau level and the Greens function equation of motion approach we have developed previously is readily adapted to the present situation. 10] For small enough $|\nu-1|$, the skyrmions can be considered to be point particles and it is known from Madelung energy calculations for 2D lattices 11] that they will form a triangular lattice. Nevertheless, we find that by the time $\nu=1.1$ the SLA state is lower than the TLF in energy by $\approx 30$ times the amount by which the Madelung energy would favor the opposite energetic ordering of the states. The self-consistent spin-textures for the TLF and SLA states at $\nu=1.1$ are illustrated in Fig. 2. The skyrmions are evidently more compact for the higher energy TLF state and this is the origin of the differences in spin-polarization between these states.

To understand the differences between SLA and TLF states in greater depth it is useful to compare the contributions to the energy per electron from exchange, Hartree and Zeeman energies as shown in Fig. 3 for $g \mu_{B} B=0.015 e^{2} / \ell$. We express our results in terms of the energy $\left(\epsilon_{q p}\right)$ increase per quasiparticle, $N_{q p}=$ $\left|N-N_{\phi}\right|$, when the quasiparticles are created at fixed $N$ by varying $N_{\phi}$ :

$$
\epsilon_{q p} \equiv \frac{\nu\left[\epsilon+\left(e^{2} / \ell\right) \sqrt{\frac{\pi}{8}}+g \mu_{B} B / 2\right]}{|1-\nu|}
$$

where $\epsilon=E / N$ is the energy per electron, $g$ is the gfactor for the 2DEG system, and $\mu_{B}$ is the electron Bohr magneton. (The Hartree, exchange, and Zeeman contributions to $\epsilon_{q p}$ are given by terms of corresponding origin on the right-hand-side of Eq. 1; $-\left(e^{2} / \ell\right) \sqrt{\pi / 8}$ is the exchange energy per-particle at $\nu=1$.) This convention for the energy per charge is implicit in the field theoretical expressions and we choose it here because it will allow our Hartree-Fock results and results obtained in the fieldtheory description to be compared directly. In the limit of vanishing skyrmion density $\epsilon_{q p}$ is the so-called neutral quasiparticle energy. 12 It follows from particle-hole symmetry that with this defintion antiskyrmion quasiparticle energies are smaller than skyrmion quasiparticle energies by $g \mu_{B} B$.

We first discuss the constituents of the energy for the Wigner crystal (WC) state which occurs near $\nu=1$ in the limit of very strong Zeeman coupling. In this state a triangular (Wigner) crystal 13] of single-reversed-spin quasiparticles is formed on the background of a full Landau level of aligned spins. The reversed spins are localized to the greatest extent possible with the lowest Landau level. For the WC state, the exchange and Hartree contributions to the energy per quasiparticle, $\epsilon_{q p}^{X}$ and $\epsilon_{q p}^{H}$, can be calculated analytically 14 in the limit of small $\nu_{q p} ; \epsilon_{q p}^{X} \rightarrow\left(e^{2} / \ell\right)\left[(\pi / 8)^{1 / 2}-(\pi / 16)^{1 / 2}\right] \approx 0.1835\left(e^{2} / \ell\right)$ and $\epsilon_{q p}^{H} \rightarrow\left(e^{2} / \ell\right)(\pi / 16)^{1 / 2} \approx 0.4431\left(e^{2} / \ell\right)$, the Hartree self-interaction energy of a lowest Landau level quantized cyclotron orbit. The exchange energy, which is minimized when all spins are parallel, is nearly constant with increasing $\nu_{q p}$ until the quasiparticles begin to overlap significantly while the Hartree energy is reduced at larger $\nu_{q p}$ due to spatial correlations of the quasiparticles. When the size of the quasiparticles $(\sim \ell$ for the WC state) is much smaller than the distance between quasiparticles the Hartree energy is reduced by the Madelung energy per particle [11]; when the quasiparticles form a triangular lattice $\epsilon_{\text {mad }} \approx-0.7821 \nu_{q p}^{1 / 2}\left(e^{2} / \ell\right)$. In Fig. 3 we have plotted the difference between the Hartree energy and the Madelung energy vs. $\nu_{q p}$. For the WC state the remaining variation is due to the fact that the electrons are localized in cyclotron orbits and not at points. The Zeeman energy contribution to $\epsilon_{q p}$ is $g \mu_{B} B\left\langle S_{q p}\right\rangle$ where $\left\langle S_{q p}\right\rangle$ is the number of reversed spins per quasiparticle. ( $\left\langle S_{q p}\right\rangle$ is smaller for antiskyrmions than for skyrmions by 1.) For the WC state $\left\langle S_{q p}\right\rangle \equiv 1$ for skyrmions and 0 for antiskyrmions.

In the absence of Zeeman coupling a skyrmion quasiparticle has lower energy than a reversed spin quasiparticle because its charge is spread out over an arbitrarily large distance, reducing $\epsilon_{q p}^{H}$ to zero while at the same time keeping spins locally nearly parallel so that $\epsilon_{q p}^{X}$ increases only 22.5] to $\left(e^{2} / \ell\right)(\pi / 32)^{1 / 2} \approx 0.3133\left(e^{2} / \ell\right)$. When a finite Zeeman coupling is present, the skyrmion quasiparticle must shrink to reduce its Zeeman energy [2,7] and the Hartree energy increases. For an isolated skyrmion $\left(\nu_{q p} \rightarrow 0\right)$ and $g \mu_{B} B=0.015 e^{2} / \ell$ we find using the approach [15] of Ref. [7] that $\epsilon_{q p}^{H}=0.2499\left(e^{2} / \ell\right)$, $\epsilon_{q p}^{X}=0.2957\left(e^{2} / \ell\right)$ and that $\left\langle S_{q p}\right\rangle=3.83$. In Fig. 3 we see that for the TLF state the Hartree energy quickly rises from the isolated skyrmion value toward the $\mathrm{WC}$ value and $\left\langle S_{q p}\right\rangle$ quickly decreases from the isolated skyrmion value to 1 as $\nu_{q p}$ increases. Evidently there is a strong short-range repulsive interaction between skyrmions in the TLF Skyrme state which quickly shrinks them into single-reversed-spin quasiparticles. The origin of this repulsion is an increase in exchange energy in the region between neighboring skyrmions with identical azimuthal orientations of $m_{\perp}$. As we see in Fig. 2 the azimuthal 
orientation of $m_{\perp}$ must then change by $\pi$ relatively abruptly along the line connecting neighboring skyrmion centers; in the field-theoretical description [2,5] the associated increase in exchange energy is described by a term $\propto\left|\nabla m_{\perp}\right|^{2}$ and the resulting repulsive interaction will be roughly proportional to $m_{\perp}^{2}$ evaluated at the mid-point between skyrmion centers. Clearly this repulsive interaction is minimized if neighboring skyrmions have opposite azimuthal orientations for $m_{\perp}$ as illustrated for the SLA state in Fig. 2. (It seems clear that the lowest energy triangular Skyrme lattice will be a three-sublattice state with $2 \pi / 3$ relative orientation but this state will, we believe, be higher in energy than the SLA state unless $\nu$ is very close to 1.) The exchange energy thus favors bipartite lattices for a Skyrme crystal. Our numerical results for the SLA state show that the skyrmions shrink relatively slowly as $\nu_{q p}$ increases; for this arrangement the Skyrme crystal is able to tolerate much tighter packing of the skyrmions and the rapid decrease in spin-polarization persists out to quite large values of $\nu_{q p}$ as shown in Fig. 1 and observed experimentally. [8]

The size of a quasiparticle in a Skyrme crystal is limited by the lattice constant of the crystal until, with decreasing $\nu_{q p}$ the lattice constant becomes substantially larger than the size of an isolated skyrmion quasiparticle. For weaker Zeeman couplings, isolated skyrmions are larger [2]7] and spin-polarization will decrease more rapidly for $\nu$ very close to $\nu=1$; for larger values of $|\nu-1|$ where the skyrmion size is limited by inter-skyrmion interactions, the spin-polarization is much less dependent on the Zeeman coupling. For example we find for the TLF state at $g \mu_{B} B /\left(e^{2} / \ell\right)=0.0015$ that $P=0.7$ at $\nu=1.03$, substantially smaller than the values reported in Fig. 1, while for $\nu=1.15, P=0.7$ again, nearly identical to Fig. 1 .

From our calculations we can conclude that for typical $g \mu_{B} B$ values, the ground state of the 2DEG near $\nu=1$ is composed of skyrmionic charged excitations of the ferromagnetic $\nu=1$ background. When these quasiparticles are sufficiently dilute they will crystallize because of the absence of kinetic energy. Although we have not completed an exhaustive survey of possible Skyrme lattices, we believe that SLA Skyrme crystal will be lowest in energy once the lattice constant is small enough for the gradient energy to overwhelm the relatively modest structural preferences of the Hartree energy. It is difficult for us to estimate the range of filling factors over which the Skyrme crystal is favored over fluid states of the 2DEG. However, it seems unlikely that the energy competitions which determines skyrmion size will be very sensitive to the existence of long-range-order. We anticipate the existence of a Skyrme liquid state, which will be lower in energy than maximally spin polarized liquid states and which will have a spin polarization quite close to that of the SLA state. The Skyrme liquid should have a typical coordination number closer to four than to six because of the dependence of the exchange energy on the relative azimuthal orientations of $m_{\perp}$ for nearby skyrmions.

The authors thank Sean Barrett, Lotfi Belkhir, Steve Girvin, Peter Littlewood, Aron Pinczuk, Shivaji Sondhi, Carlos Tejedor, and Kun Yang for helpful discussions. This work was supported in part by NATO CRG No. 930684, by the NSF through Grants Nos. DMR 92-02255 and DMR 94-16906, by the CICyT of Spain under Contract No. MAT 94-0982, by NSERC of Canada, and by FCAR from the Government of Quebec. HAF acknowledges the support of the A.P.Sloan Foundation and the Research Corporation. LB thanks AT\&T-Bell Labs at Murray Hill and AHM thanks UAM for hospitality during the realization of this work.

[1] The Quantum Hall Effect, edited by R.E.Prange and S.M.Girvin (Springer-Verlag, New York, 1990).

[2] S.L.Sonhdi, A.Karlhede, S.A.Kivelson, and E.H.Rezayi, Phys.Rev.B 47, 16419 (1993).

[3] E.H. Rezayi, Phys. Rev. B 36, 5454 (1987); Phys. Rev. B 43, 5944 (1991).

[4] D.H.Lee and C.L.Kane, Phys.Rev.Lett. 64, 1313 (1990).

[5] K.Moon, H.Mori, Kun Yang, S.M.Girvin, A.H.MacDonald, L.Zheng, D.Yoshioka, and Shou-Cheng Zhang, Phys. Rev. B 51, 5138 (1995).

[6] R.Rajaraman, Solitons and Instantons (North-Holland, Amsterdam, 1989).

[7] H.A.Fertig, L.Brey, R.Côté and A.H.MacDonald, Phys. Rev. B 50, 11018 (1994).

[8] S.E.Barret, G.Dabbagh, L.N.Pfeiffer, K.West and R.Tycko, Phys. Rev. Lett., to appear (1995).

[9] I.Klebanov, Nucl.Phys. B 262, 133 (1985); L.Castillejo et al, Nucl.Phys. A 501, 801 (1989); M.Kugler et al, Phys.Rev.D 40, 3421 (1989).

[10] R.Côté and A.H.MacDonald, Phys.Rev.B 44, 8759 (1991) ; R.Côté, L.Brey and A.H.MacDonald, Phys.Rev.B 46, 10239 (1992); R.Côté, L.Brey, H. Fertig, and A.H.MacDonald, Phys.Rev.B to appear (1995). Technical details of the present calculations will be published elsewhere.

[11] Lynn Bonsall and A.A. Maradudin, Phys. Rev. B 15, 1959 (1977).

[12] R.Morf and B.I. Halperin, Phys. Rev. B 33, 2221 (1986); A.H. MacDonald, and S.M. Girvin, Phys. Rev. B 34, 5639 (1986).

[13] See for example A.H. MacDonald in Low Dimensional Electronic Systems, edited by Günther Bauer, Friedemar Kuchar, and Helmut Heinrich (Springer-Verlag, Berlin, 1993) p. 189.

[14] A.H. MacDonald, unpublished.

[15] Since the $\hat{z}$-component of the total spin is a good quantum number, for an isolated skyrmion $\left\langle S_{z}\right\rangle$ is an integer in the exact many-body ground state. The Hartree-Fock estimate is a continuous function of system parameters and applies unless $k_{B} T$ is smaller than energetic separa- 
tion between single-skyrmion states with adjacent values of $\left\langle S_{z}\right\rangle$. For typical systems we do not expect the discreteness of the allowed values for $\left\langle S_{z}\right\rangle$ to be observable above $\sim 1 \mathrm{~K}$. In the Skyrme crystal skyrmion-skyrmion interactions will cause $\left\langle S_{z}\right\rangle$ to fluctuate on each site even at $T=0$.

FIG. 1. Variation of the spin polarization, $P$, as a functions of $\nu$, for SLA and TLF Skyrme crystal states and for different values of $g^{*} \equiv g \mu_{B} B /\left(e^{2} / \ell\right)$. The open circles are experimental data obtained for $g^{*}$ near 0.016 , while the filled circles show data obtained for $g^{*}$ near 0.021 . The spin polarization in the WC state is the same as that for independent electrons.

FIG. 2. Two-dimensional vector representation of the $x-y$ components of the spin density of a crystal of skyrmions as obtained in the HFA. The length of the arrows is proportional to the local magnitude of $m_{\perp}$ and their direction indicates the local orientation. The magnitude of $m_{\perp}$ is small for $m_{z}$ near -1 , near the center of a skyrmion, and for $m_{z}$ near +1 , outside of a skyrmion.(a) TLF state at $\nu=1.1$ and $g^{*}=0.015$; (b) SLA state at $\nu=1.1$ and $g^{*}=0.015$.

FIG. 3. Zeeman (a), Hartree (b) and exchange (c) contributions to the energy per quasiparticle for the Wigner crystal state $\left(^{*}\right)$ and for the TLF (triangles) and SLA (squares) Skyrme crystal states. The results for $\nu=1$ were obtained analytically for the WC state and from isolated skyrmion calculations for the Skyrme crystal states. The Zeeman energy is expressed in terms of the average number of reversed spins per quasiparticle and the Madelung contribution has been subtracted from the Hartree contribution, as discussed in the text. 